\title{
Toward a Systems Analysis of the Root
}

\author{
P.N. BENFEY \\ Department of Biology, Duke Center for Systems Biology, Duke University, \\ Durham, North Carolina 27708 \\ Correspondence: philip.benfey@duke.edu
}

\begin{abstract}
During the past 20 years, our work on root development has been influenced by and has contributed to three biological approaches: molecular genetics, genomics, and systems biology. Characterization of mutations that affect root radial patterning led to the identification of a transcription factor that acts as both a signaling molecule and a key developmental regulator. Combining cell sorting with microarray analysis provided a platform for determining genome-wide expression profiles of mRNAs under standard and stress conditions, revealing a vast amount of tissue-specific response. A focus on connections among molecular components identified a tissue-specific gene regulatory network and a clock-like process that determines the position of lateral roots along the primary root axis. Finally, the genetic basis for the physical network of different roots that constitutes root system architecture is being dissected using automated imaging of growing root systems.
\end{abstract}

\section{THREE REVOLUTIONS}

I feel that I have been very fortunate in having benefited from and participated in three transformations in biology, for which the word "revolution" does not seem farfetched. When I was a graduate student in the early 1980s, the tools and approaches of molecular genetics were being introduced. The primary goal was to identify a gene underlying a phenotype by use of recombinant DNA technology. Once a gene was identified, the next steps were to find what it acted upon and what acted upon it. The result was usually described as a linear pathway.

In the 1990s, the first complete genomes of higher organisms were completed. The promise of the genomic revolution was that knowledge of the entire parts list of an organism would provide deep insights into the underlying biology. One approach was to compare the genomes or the output of the genomes, for example the mRNAs, between organisms, treatments, developmental stages, or disease states. The results were generally given as a list of specific genes or transcripts.

The beginning of the new millennium saw a certain amount of disenchantment. With all that had been learned about pathways from molecular genetics and even with the parts lists of genomics and the attendant comparisons, there remained a persistent inability to accurately predict phenotype from genotype.

At about the same time, there was a realization that perhaps what was missing was sufficient understanding of how molecular components interacted. From knowledge of these connections, the thinking went, we might discover biological processes that were opaque when we read lists of genes or looked at linear pathways. Examples of "emergent properties" are circadian rhythm or cell cycle control, which results from the particular types of molecular interactions and the topologies of the networks that connect them. The field of "systems biology" focuses on the connections between molecular components and the dynamics of the information flow through the resulting networks.

My goal is to place some of the work from my laboratory during the last two decades in the context of these three revolutions. I begin with our efforts to understand the patterning of root radial organization, then discuss how we exploited the simplifying aspects of root development to make genome-wide expression profiles at celltype-specific resolution, and finish with our ongoing efforts to find interactions and understand the dynamics of root development.

\section{PATHWAYS TO RADIAL PATTERNING}

I first began to appreciate the simplifying aspects of root anatomy during the dissection of the CaMV $35 \mathrm{~S}$ promoter, which I performed while a postdoc in Nam-Hai Chua's laboratory. My approach was to fuse different portions of the $35 \mathrm{~S}$ promoter to the GUS reporter and then look for expression in sections of tobacco tissues (Benfey et al. 1989, 1990a,b; Benfey and Chua 1990). Several of the constructs gave cell-type-specific expression in the root and I was struck by the fact that median longitudinal sections gave access to nearly all of the root cell types. This was due to the organization of root tissues as a series of concentric cylinders surrounding the internal vascular tissue. Another major simplifying aspect of root anatomy is that there is a stem cell center at the tip of the root and the progeny of the four different stem cell types remain aligned with the stem cell. This makes it trivial to determine the relative age of a cell because the youngest cell is adjacent to the stem cell, the next youngest is adjacent to that cell, etc.

To apply molecular genetics to root development, I moved from tobacco to Arabidopsis and carried out a very simple genetic screen in which I looked for mutants 
with shorter or fatter roots. The primary reason for this screen was that it was much easier to spot a short or fat root than a thin or long root. I was fortunate that Ken Feldmann allowed me access to the collection of insertional mutants he had made at DuPont. From that initial screen, I identified a mutant with shorter roots, which I very creatively named "short-root" (Benfey et al. 1993). A collaboration with Keith Roberts helped identify a more salient defect: The short-root mutant was missing one of its cell layers. Between the epidermis and pericycle, there are normally two ground tissue layers, called the cortex and endodermis. In short-root, there was only a single layer. At about the same time, we found another mutant with shorter roots that we determined was also missing a cell layer between the epidermis and pericycle. Because it was able to complement short-root and thus the mutation was in a different gene, we named it scarecrow after the character in the Wizard of $\mathrm{Oz}$ who is missing a brain (Benfey et al. 1993). Although both mutations resulted in the loss of a cell layer, when we analyzed markers that were specific to endodermis or cortex we discovered an interesting difference. The short-root mutation results in the total loss of endodermal markers, whereas in the scarecrow mutant, the remaining cell layer has differentiation markers of both endodermis and cortex. This suggested to us that SCARECROW is primarily involved in the cell division process per se, because specification of the two cell types takes place in the mutant, whereas SHORT-ROOT has a role in both the asymmetric cell division and specification of endodermis. We hypothesized that SHORT-ROOT could act through SCARECROW to mediate asymmetric cell division.

Teams led by Laura DiLaurenzio and Yka Helariutta cloned the mutated genes for SCARECROW (SCR) (Di Laurenzio et al. 1996) and SHORT-ROOT (SHR) (Helariutta et al. 2000), respectively. The genes turned out to be related and among the founding members of a plant-specific family of transcription factors that we named the GRAS family (Pysh et al. 1999). The expression pattern of $S C R$ was as we expected - confined to the stem cell that gives rise to the ground tissue and remaining on in the endodermal cell layer. The surprise came when we determined the expression pattern of SHR. Its mRNA was found solely in the vascular tissue and pericycle, which together comprise the stele. How then did it regulate the division of the ground tissue stem cell into endodermis and cortex, and how did it control endodermal specification? The answers came when we determined the expression pattern of the SHR protein. Keiji Nakajima and Giovanni Sena found that, in addition to being found in the stele, SHR was also localized to the cortex/endodermal initial (CEI) and its progeny (Nakajima et al. 2001). There was also a striking difference in its subcellular localization because it was found in both the cytoplasm and nucleus in the stele, but primarily in the nucleus in the endodermis and stem cells. These findings provided the first evidence that SHR physically moves among cell layers, probably through the plant-specific junctions called plasmodesmata.

Further work over many years resulted in a detailed understanding of the SHR/SCR network. Hongchang
Cui showed that SHR and SCR directly interact and that this interaction prevents SHR from moving beyond the endodermis (Cui et al. 2007). Kim Gallagher performed a structure/function analysis on the SHR protein and identified residues that were essential for movement (Gallagher et al. 2004). Work from Mitch Levesque, Teva Vernoux, and Hongchang Cui demonstrated that SHR and SCR bind to chromatin and identified direct and indirect downstream targets (Levesque et al. 2006). A key finding was that SHR and SCR bind in similar locations to the promoters of a number of genes, suggesting that they may bind as a heterodimer or larger-order complex (Cui et al. 2007). More recently, Rosangela Sozzani showed that SHR and SCR directly activate a cyclin, CYCD6, that has a central role in asymmetric cell division of the CEI daughter (Sozzani et al. 2010). In a collaboration with two former postdocs, Yka Helariutta and Ji-Young Lee, along with Yka's former postdoc, Annelie Carlsbecker, we discovered that SHR and SCR activate expression of a microRNA in the endodermis that then moves into the vascular tissue to form a gradient that regulates xylem identity (Carlsbecker et al. 2010). Thus, after 20 years of investigation, this pathway is still yielding important insights into the patterning processes taking place in higher plants.

\section{PROFILING THE ROOT}

Even before genomic sequences became available, there were various techniques to compare mRNAs between different tissues or treatments. I was initially very skeptical of microarray technology because it was not evident to me how it would differ from approaches such as differential display or subtractive hybridization. The outcome of those techniques was a list of differentially expressed mRNAs, but there had been a paucity of cases in which important genes were identified using these approaches. My skepticism turned out to be misplaced, because the capacity of microarrays to interrogate the entire genome dramatically increased their utility when compared with the previous techniques. The challenge then became how to use microarrays to address questions in plant development. Our previous work on the root made us appreciate how different were the various root cell types. To grind up whole roots for their RNA would result in the loss of cell-type-specific information.

For a while, we explored the feasibility of performing high-throughput in situ hybridizations, but the lack of a reliable whole-mount process and a failed grant application convinced us to look for alternatives. Ken Birnbaum decided to try cell sorting as a means to profiling individual cell types. In collaboration with David Galbraith, who was the world's expert on sorting plant cells, we worked out the protocol for enzymatically digesting the cell walls of Arabidopsis roots that carried a cell-typespecific GFP marker, then sorting for the GFP-expressing cells. Once these cells were sorted, RNA was isolated and used for hybridization to Affymetrix microarrays (Birnbaum et al. 2003). There were many reasons why this 
approach should not have worked, the primary one being that during the course of cell wall digestion and passage through the cell sorter, the mRNA profiles could change. To reduce this likelihood, we performed the digestion and sorting as rapidly as possible. Under these conditions, we detected changes attributable to the procedure, but they were relatively minor. Another potential issue was that with our rapid procedure, the number of cells liberated from each digested root was relatively small. We were concerned that this might result in a skewed representation of cell types or developmental stages. As far as we were able to observe, this was not the case.

Ken Birnbaum performed an initial analysis of five cell types. To profile along the longitudinal axis of the root, which represents a developmental timeline, we did not have appropriate GFP markers. Instead, we cut roots at easily discernible developmental landmarks, for example, the transition from cell division to cell expansion, and combined the pieces from multiple roots for RNA extraction and hybridization to microarrays. The results demonstrated a striking amount of cell-type- and developmental-zone-specific gene expression, strongly suggesting that analyses of entire organs, such as roots or leaves, dilute out a lot of important information (Birnbaum et al. 2003). Having validated the approach, Siobhan Brady led a team that performed a comprehensive analysis across 19 cell populations (Brady et al. 2007). To increase the resolution along the longitudinal axis, she cut a single root into 12 pieces and extracted RNA from the individual pieces. A replicate root was analyzed in the same manner. David Orlando was instrumental in developing computational tools to analyze the massive amount of data. He identified coregulated expression clusters in both cell type and developmental zone data. The major surprise from this analysis was the discovery of a large number of fluctuating expression patterns along the longitudinal axis. These consisted of coregulated genes whose expression peaked at an early stage of development and then rose again at a later stage of development (Brady et al. 2007). We normally think of development as a progressive, unidirectional process; thus, gene expression that appeared to be repetitive was unexpected-all the more so in that 17 of the 41 dominant coregulated expression patterns exhibited repetitive fluctuations. This highlights the value of this type of "discovery-driven," as opposed to "hypothesis-driven," research.

Once we had a comprehensive map of gene expression for plants grown under normal laboratory conditions, we decided to discover what happens when plants are subjected to stress. There is ample evidence that plants are exquisitely responsive to external stimuli, which is a good reason for growing them in highly controlled environments. However, most of the previous work had analyzed the response to stress at the level of entire organs (e.g., leaves or roots) or for the entire plant. Jose Dinneny decided to determine whether increased salt would differentially affect gene expression across cell types or developmental stages. This was a bold move because excess salt is toxic to cells, suggesting that all cells might respond in a similar fashion to try to reduce the toxic levels. What Jose found was that growth on high salt causes a massive amount of cell-type- and developmental-zone-specific expression (Dinneny et al. 2008). A similar result was found by Terri Long looking at the response to growth on low iron (Dinneny et al. 2008; Long et al. 2010). Differential responses at the level of cell types or developmental zones appear to be part of a strategy for the root to acclimate itself to changes in its environment. More recently, Anjali Iyer-Pascuzzi and Terry Jackson extended this analysis to low $\mathrm{pH}$ and sulfur deficiency (Iyer-Pascuzzi et al. 2011). A comparison across all available stress-responsive data sets suggested that there is no universal stress response in the root.

Another use for RootMap data was to look for genes whose expression pattern suggested that they might be involved in a specific developmental process. Hironaka Tsukagoshi decided to focus on the transition from rapid cell division to cell elongation, which is the first step toward differentiation. He identified 50 genes that were activated at the beginning of the elongation process and then decreased in expression as elongation ended. Insertional mutants for all 50 genes were tested for an effect on root growth, and one was identified that actually caused roots to grow faster than normal (Tsukagoshi et al. 2010). The gene, which we named UPBEAT1, was introduced into plants behind a strong constitutive promoter, causing a reduction in root growth. In collaboration with Wolfgang Busch, Hiro used microarrays and DNA binding assays to identify a set of peroxidases as direct downstream targets of UPBEAT1. He then showed that these peroxidases regulate the level of reactive oxygen species in the elongation zone and that the level and type of reactive oxygen species causes changes in growth. Thus, starting with coexpression data, we were able to identify a novel mechanism controlling root growth.

Although there are almost certainly anomalies in these cell-type- and developmental-zone-specific data sets due to the mode of preparation and sorting, to date no alternative method has appeared that is as broadly applicable. In addition to determining the levels of mRNAs, we have used the cell-sorting approach to identify cell-type-specific small RNAs (Breakfield et al. 2012), proteins (Petricka et al. 2012), and metabolites (Rogers et al. 2012). We are currently using it to perform an updated "RootMap" of RNA using RNA-seq with the goal of profiling the more than 6000 genes that are not represented on the Affymetrix microarray. In addition, we will be able to assess for the first time the extent of differential splicing that occurs in a cell-type-specific manner. A third objective is to identify noncoding RNAs that are differentially expressed. Thus, the cell-sorting approach continues to provide a wealth of information regarding molecules that differ in their concentration between cell types.

\section{EMERGENT PROPERTIES OF ROOT NETWORKS}

When I first started to read about systems biology, I contextually came across the term "emergent properties." 
I really did not understand how this applied to biology and found it to be somewhat mystical. Only later did I find a useful example in the behavior of bird flocks. No matter how intensively one studies an individual bird, one would never understand flock behavior. The key is the connection between birds - how one bird reacts to the birds around it. Thus, the behavior of a flock is an emergent property of the connections among birds. I find this to be a useful analogy for considering aspects of biology. The properties of individual components are important to understand, but when they are linked together in a network, new behaviors can emerge. There are two important steps to applying this understanding to biology; the first is to identify who is connected to whom and the second is to determine how the network functions.

Our first attempt at identifying network connections in a comprehensive manner was led by Siobhan Brady. Her goal was to determine the regulatory connections among transcription factors in the stele. Her starting point was the RootMap from which she identified all of the transcription factors that were enriched in the stele. Then, in collaboration with Marian Walhout, Siobhan used the yeast one-hybrid assay to determine those transcription factors that were able to bind to the promoters of the other stele-enriched transcription factors (Brady et al. 2011). In this manner, she was able to place approximately one-quarter of the transcription factors in a network. To validate the network, she analyzed the effects of mutations in the putative upstream factors on their supposed downstream targets. Because many of the target promoters were bound by multiple transcription factors, our expectation was that most mutations would have little observable effect. The surprise was that $>65 \%$ actually did have a reproducible effect on the downstream target. Moreover, we were then able to determine if the upstream regulator was an activator or a repressor. In collaboration with Sebastian Ahnert, we used the strengths of activation or repression to develop a model that predicted the relative importance of each of the upstream factors in network function (Brady et al. 2011).

We discovered another type of emergent behavior when we asked the question "How are lateral roots positioned along the primary root?" Looking at the roots of Arabidopsis, one would be excused for thinking there must be a periodic process that determines their relative position, because they appear to form at regular intervals along the root. Yet, according to the textbooks, lateral roots form at random positions and, once formed, they inhibit other lateral roots from initiating nearby. Thus, their apparent periodicity is the byproduct of random initiation and lateral inhibition.

The first clue that there might be another explanation came from a study by Ive De Smet in Tom Beeckman's laboratory. Ive observed that when he lightly stained seedlings containing the DR5-GUS auxin reporter, he found that the number of roots that stained in a specific region of the meristem appeared to oscillate depending on the length of time that they had grown (De Smet et al. 2007). He astutely hypothesized that this oscillation was related to the positioning of lateral roots, because they appeared to form with a similar periodicity. In my laboratory, Miguel Moreno-Risueno wanted to observe this oscillation in real time. To do so, he generated a reporter with the DR5 promoter driving firefly luciferase. Imaging of this reporter construct revealed oscillations with a period of $\sim 6 \mathrm{~h}$ as well as expression that appeared after each oscillation and remained as a series of illuminated spots along the length of the root (Moreno-Risueno et al. 2010). Miguel then observed that these points were precisely where lateral roots form and thus named them "prebranch sites." He and Jaimie Van Norman demonstrated that this oscillatory process was buffered against changes in the rate of root growth, suggesting that it had features of a biological clock; thus, they named it the "lateral root clock."

Because DR5 is widely used as a reporter for auxin activity, the first hypothesis was that auxin concentration or activity was fluctuating periodically in the region of the root exhibiting this oscillation. If this were the case, one would expect other auxin-responsive genes expressed in this region to show a similar oscillatory pattern. Miguel identified one such auxin-responsive gene, IAA19, made a luciferase reporter construct with its promoter, and showed that it had a dose-response curve very similar to that of DR5 for exogenously applied auxin. However, when its expression was imaged over time, he did not observe oscillatory behavior. If not auxin, then what is driving the DR5 oscillation? Identification of other genes oscillating in this region of the root might provide clues as to how this oscillation works. Miguel came up with a scheme to do just that: He cut two sections from the region he called the oscillation zone $(\mathrm{OZ})$ from 40 individual seedlings containing the DR5-GUS construct. By quantitative reverse-transcriptase-polymerase chain reaction (qRT-PCR) analysis, he determined the level of GUS mRNA in each section and used this to order the roots along the oscillation wave. He then performed a microarray analysis on the individual sections from 20 roots. To identify periodic expression patterns, he used two mathematical methods, one based on a Fourier transform approach that assumes periodic behavior and the other on an information theoretic approach that does not assume periodic behavior but looks for patterns. There was remarkable overlap in the genes identified by the two approaches, and we identified $\sim 2000$ genes appearing to oscillate in phase with DR5 and another 1400 genes appearing to oscillate in antiphase with DR5. Several promoters from oscillating genes that encoded transcription factors were fused to luciferase and introduced into plants. Examples of oscillation both in phase and in antiphase with DR5 were observed from these promoters. To determine whether these genes are involved in lateral root formation, Miguel screened insertional mutations in these genes. He found that single mutations had fairly subtle effects, but that double and triple mutants exhibited dramatic reductions in the number of lateral roots and prebranch sites (Moreno-Risueno et al. 2010). Our current hypothesis is that networks of transcription factors are configured in such a way as to generate oscillations in expression and that this emergent behavior establishes the periodic positioning of lateral roots. 
The formation of lateral roots is one aspect of root system architecture - the ensemble of roots through which a plant explores and exploits its subterranean environment. Root system architecture can also be regarded as an emergent property of the number and types of roots present, their individual growth rates, and the angles at which they grow. This is a highly complex system that normally grows in a very complex environment, which includes soil, microbes, fungi, and invertebrates. It is, no doubt, the combination of complex environment and opaque substrate that have worked together to limit our understanding of the genetic basis of root system architecture. In the face of this complexity, we decided to take a reductionist approach with the goal of performing threedimensional phenotyping of root system architecture. Initially working with rice, we grew plants in cylinders with nutrients and translucent gellan gum, which allowed the root systems to form in three dimensions. We placed the cylinders on a turntable connected to a computer-controlled camera that allowed us to perform tomographic reconstructions. A team led by Anjali Iyer-Pascuzzi and Chris Topp imaged the roots of a large number of rice cultivars. In collaboration with Joshua Weitz, John Harer, and Herbert Edelsbrunner, they developed mathematical ways of quantifying aspects of the two- and three-dimensional root system architecture. They then showed that these mathematical descriptors could be used to distinguish among cultivars for which the root systems are indistinguishable by the human eye (Iyer-Pascuzzi et al. 2010). This gave us the confidence to analyze a recombinant inbred line population developed by Adam Price in which the parents have very different root system architectures. The preliminary analysis of the quantitative trait loci (QTL) looks very promising, suggesting that we may be able to identify genes underlying the emergent properties of root system architecture.

\section{CONCLUSIONS AND FUTURE DIRECTIONS}

Although the three revolutions have led to important new insights in biology, their importance for plant biology has seemed smaller than has their role in improving human health. A concurrence of events is changing this perception. The major challenges facing the world todayfeeding a rapidly growing population, finding sustainable sources of energy, and dealing with a changing climateall relate directly to plant biology. Moreover, plant biology is well positioned to address the fundamental biological question of how genotype maps to phenotype. In our own work, we see an increasing role for image-based realtime analysis to better understand the emergent properties of networks such as that of SHR and SCR controlling stem cell divisions and subsequent differentiation or to follow the formation of the physical networks of root system architecture. As we gain an understanding of the dynamics of networks, we may find ourselves in the next revolution, which allow us to integrate information across the levels of biological organization from molecule to cell to organism to population to ecosystem.

\section{ACKNOWLEDGMENTS}

I thank all of the graduate students and postdocs who have contributed immensely to our research during the last 20 years. I have only been able to highlight a small number of the many contributions made by this very special group of people. The work described in this paper was funded by the National Institutes of Health grants RO1-GM43778 and P50-GM081883, the National Science Foundation programs Arabidopsis 2010 and PGRP, and by the Defence Advanced Research Project agency.

\section{REFERENCES}

Benfey PN, Chua NH. 1990. The cauliflower mosaic virus 35S promoter: Combinatorial regulation of transcription in plants. Science 250: 959-966.

Benfey PN, Ren L, Chua NH. 1989. The CaMV 35S enhancer contains at least two domains which can confer different developmental and tissue-specific expression patterns. $E M B O$ J 8: 2195-2202.

Benfey PN, Ren L, Chua NH. 1990a. Tissue-specific expression from CaMV $35 \mathrm{~S}$ enhancer subdomains in early stages of plant development. EMBO J 9: 1677-1684.

Benfey PN, Ren L, Chua NH. 1990b. Combinatorial and synergistic properties of CaMV $35 \mathrm{~S}$ enhancer subdomains. EMBO J 9: $1685-1696$.

Benfey PN, Linstead PJ, Roberts K, Schiefelbein JW, Hauser MT, Aeschbacher RA. 1993. Root development in Arabidopsis: Four mutants with dramatically altered root morphogenesis. Development 119: 57-70.

Birnbaum K, Shasha DE, Wang JY, Jung JW, Lambert GM, Galbraith DW, Benfey PN. 2003. A gene expression map of the Arabidopsis root. Science 302: 1956-1960.

Brady SM, Orlando DA, Lee JY, Wang JY, Koch J, Dinneny JR, Mace D, Ohler U, Benfey PN. 2007. A high-resolution root spatiotemporal map reveals dominant expression patterns. Science 318: $801-806$.

Brady SM, Zhang L, Megraw M, Martinez NJ, Jiang E, Yi CS, Liu W, Zeng A, Taylor-Teeples M, Kim D, et al. 2011. A stele-enriched gene regulatory network in the Arabidopsis root. Mol Syst Biol 7: 459.

Breakfield NW, Corcoran DL, Petricka JJ, Shen J, Sae-Seaw J, Rubio-Somoza I, Weigel D, Ohler U, Benfey PN. 2012. Highresolution experimental and computational profiling of tissuespecific known and novel miRNAs in Arabidopsis. Genome Res 22: 163-176.

Carlsbecker A, Lee JY, Roberts CJ, Dettmer J, Lehesranta S, Zhou J, Lindgren O, Moreno-Risueno MA, Vaten A, Thitamadee $S$, et al. 2010. Cell signalling by microRNA165/6 directs gene dose-dependent root cell fate. Nature 465: 316321.

Cui H, Levesque MP, Vernoux T, Jung JW, Paquette AJ, Gallagher KL, Wang JY, Blilou I, Scheres B, Benfey PN. 2007. An evolutionarily conserved mechanism delimiting SHR movement defines a single layer of endodermis in plants. Science 316: 421-425.

De Smet I, Tetsumura T, De Rybel B, Frey NF, Laplaze L, Casimiro I, Swarup R, Naudts M, Vanneste S, Audenaert D, et al. 2007. Auxin-dependent regulation of lateral root positioning in the basal meristem of Arabidopsis. Development 134: $681-690$.

Di Laurenzio L, Wysocka-Diller J, Malamy JE, Pysh L, Helariutta Y, Freshour G, Hahn MG, Feldmann KA, Benfey PN. 1996. The SCARECROW gene regulates an asymmetric cell division that is essential for generating the radial organization of the Arabidopsis root. Cell 86: 423-433.

Dinneny JR, Long TA, Wang JY, Jung JW, Mace D, Pointer S, Barron C, Brady SM, Schiefelbein J, Benfey PN. 2008. Cell 
identity mediates the response of Arabidopsis roots to abiotic stress. Science 320: $942-945$.

Gallagher KL, Paquette AJ, Nakajima K, Benfey PN. 2004. Mechanisms regulating SHORT-ROOT intercellular movement. Curr Biol 14: 1847-1851.

Helariutta Y, Fukaki H, Wysocka-Diller J, Nakajima K, Jung J, Sena G, Hauser MT, Benfey PN. 2000. The SHORT-ROOT gene controls radial patterning of the Arabidopsis root through radial signaling. Cell 101: 555-567.

Iyer-Pascuzzi AS, Symonova O, Mileyko Y, Hao Y, Belcher H, Harer J, Weitz JS, Benfey PN. 2010. Imaging and analysis platform for automatic phenotyping and trait ranking of plant root systems. Plant Physiol 152: 1148-1157.

Iyer-Pascuzzi AS, Jackson T, Cui H, Petricka JJ, Busch W, Tsukagoshi H, Benfey PN. 2011. Cell identity regulators link development and stress responses in the Arabidopsis root. Dev Cell 21: 770-782.

Levesque MP, Vernoux T, Busch W, Cui H, Wang JY, Blilou I, Hassan H, Nakajima K, Matsumoto N, Lohmann JU, et al. 2006. Whole-genome analysis of the SHORTROOT developmental pathway in Arabidopsis. PLoS Biol 4: e143.

Long TA, Tsukagoshi H, Busch W, Lahner B, Salt DE, Benfey PN. 2010. The bHLH transcription factor POPEYE regulates response to iron deficiency in Arabidopsis roots. Plant Cell 22: $2219-2236$.
Moreno-Risueno MA, Van Norman JM, Moreno A, Zhang J, Ahnert SE, Benfey PN. 2010. Oscillating gene expression determines competence for periodic Arabidopsis root branching. Science 329: 1306-1311.

Nakajima K, Sena G, Nawy T, Benfey PN. 2001. Intercellular movement of the putative transcription factor SHR in root patterning. Nature 413: 307-311.

Petricka JJ, Schauer MA, Megraw M, Breakfield NW, Thompson JW, Georgiev S, Soderblom EJ, Ohler U, Moseley MA, Grossniklaus U, et al. 2012. The protein expression landscape of the Arabidopsis root. Proc Natl Acad Sci 109: 6811-6818.

Pysh LD, Wysocka-Diller JW, Camilleri C, Bouchez D, Benfey PN. 1999. The GRAS gene family in Arabidopsis: Sequence characterization and basic expression analysis of the SCARECROW-LIKE genes. Plant J 18: 111-119.

Rogers ED, Jackson T, Moussaieff A, Aharoni A, Benfey PN. 2012. Cell type-specific transcriptional profiling: Implications for metabolite profiling. Plant J 70: 5-17.

Sozzani R, Cui H, Moreno-Risueno MA, Busch W, Van Norman JM, Vernoux T, Brady SM, Dewitte W, Murray JA, Benfey PN. 2010. Spatiotemporal regulation of cell-cycle genes by SHORTROOT links patterning and growth. Nature 466: $128-132$.

Tsukagoshi H, Busch W, Benfey PN. 2010. Transcriptional regulation of ROS controls transition from proliferation to differentiation in the root. Cell 143: 606-616. 


\section{$\$_{\text {CSH\& }}^{\infty}$ Cold Spring Harbor Symposia SYMPOSIA}

\section{Toward a Systems Analysis of the Root}

P.N. Benfey

Cold Spring Harb Symp Quant Biol 2012 77: 91-96 originally published online December 12, 2012

Access the most recent version at doi:10.1101/sqb.2012.77.014506

$\begin{array}{ll}\text { References } & \begin{array}{l}\text { This article cites } 27 \text { articles, } 12 \text { of which can be accessed free at: } \\ \text { http://symposium.cshlp.org/content/77/91.full.html\#ref-list-1 }\end{array}\end{array}$

License

Email Alerting Receive free email alerts when new articles cite this article - sign up in

Service the box at the top right corner of the article or click here. 